\title{
A Correction for IUE UV Flux Distributions from Comparisons with CALSPEC
}

\author{
Ralph C. Bohlin ${ }^{1}$, and Luciana Bianchi ${ }^{2}$
}

\begin{abstract}
A collection of spectral energy distributions (SEDs) is available in the Hubble Space Telescope (HST) CALSPEC database that is based on calculated model atmospheres for pure hydrogen white dwarfs (WDs). A much larger set $(\sim 100,000)$ of UV SEDs covering the range $(1150-3350 \AA)$ with somewhat lower quality are available in the IUE database. IUE low-dispersion flux distributions are compared with CALSPEC to provide a correction that places IUE fluxes on the CALSPEC scale. While IUE observations are repeatable to only $4-10 \%$ in regions of good sensitivity, the average flux corrections have a precision of $2-3 \%$. Our re-calibration places the IUE flux scale on the current UV reference standard and is relevant for any project based on IUE archival data, including our planned comparison of GALEX to the corrected IUE fluxes. IUE SEDs may be used to plan observations and cross-calibrate data from future missions, so the IUE flux calibration must be consistent with HST instrumental calibrations to the best possible precision.
\end{abstract}

Subject headings: stars: atmospheres - stars: fundamental parameters - techniques: spectroscopic - ultraviolet: stars

\section{Introduction}

Flux values in physical units for astronomical objects are required to make comparisons to physical models, for example measuring relative fluxes of redshifted supernovae Ia's and, thus, in determining the nature of the dark energy that is driving the observed accelerating cosmic expansion (Scolnic et al. 2014). A good set of primary and secondary absolute flux standards is in the CALSPEC1 1 archive, where the flux calibration relies on pure hydrogen white dwarf (WD) models for G191B2B, GD153, and GD71 to calibrate HST/STIS spectrophotometry and to establish UV/optical/near-IR flux standards (Bohlin 2014).

The IUE spacecraft was launched 1978 January 26 and was sensitive in each of two UV channels

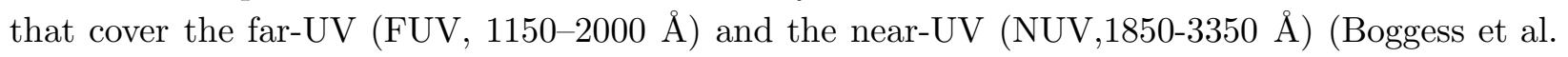

\footnotetext{
${ }^{1}$ Space Telescope Science Institute, 3700 San Martin Drive, Baltimore, MD 21218, USA

${ }^{2}$ Department of Physics and Astronomy, The Johns Hopkins University, Baltimore, MD 21218, USA

${ }^{1}$ http://www.stsci.edu/hst/observatory/crds/calspec.html
} 
1978). Each spectroscopic channel had a low-resolution and an echelle high-resolution mode with a prime and a redundant camera for each channel, i.e. SWP and SWR in the FUV and LWP and LWR in the NUV. Because of better photometric repeatability and higher sensitivity, the low dispersion modes of SWP, LWP, and LWR with resolution $\mathrm{R} \sim 6 \AA$ are of interest here. The shortwavelength redundant camera SWR was not used for many science observations. Each camera could obtain spectra with either a large aperture $(\mathrm{L})$ of $\sim 10 \mathrm{x} 20^{\prime \prime}$ or a small aperture $(\mathrm{S})$ of $\sim 3^{\prime \prime}$ diameter. The L-aperture data are photometrically repeatable, while the S-aperture transmits $20-70 \%$ of the incident light but does provide useful spectral energy distributions (SEDs) when normalized to the L-aperture.

The IUE database represents a homogenous collection of 105,584 spectra, 56227 in the Short-

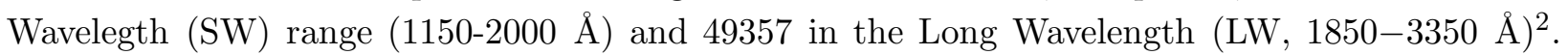
Although surpassed in quality by UV spectral data from HST instruments such as FOS, HRS, and STIS (Bohlin et al. 2001), the IUE spectral collections remain a frequently used resource with extensive sets of spectroscopic UV extinction curves and the first reference atlases of UV spectra of stars (Wu et al. 1996) and galaxies (Kinney et al. 1996). One of our projects (in preparation) exploits the IUE database to revisit the calibration of the GALEX spectra by comparing the overlapping target sample. GALEX spectra have a similar wavelength range, 1350$2830 \AA$, and slightly lower resolution and S/N than the IUE low-dispersion modes. However, the GALEX spectral database constitutes a fainter UV spectral collection than the IUE set. Because the IUE faint limits are close to the GALEX bright flux safety limits, there is a small overlap of the two datasets, which can be used to place the two benchmark spectral collections onto a consistent flux scale.

Section 2 compares the HST/STIS UV spectrophotometry to the set of stars in common with IUE to establish the corrections needed to bring IUE fluxes onto the CALSPEC scale and to estimate the precision of IUE. Section 3 describes the problematic cases, while Section 4 details the electronic access to our results. In preparation are companion papers that compare CALSPEC and IUE SEDs with a set of matched GALEX spectral SEDs and that present the entire database of GALEX grism flux distributions.

\section{Comparision of IUE and CALSPEC}

The IUE data analyzed here are extracted from the Mikulski Archive for Space Telescopes (MAST) 3 . This Archive contains both the original IUE data products from the IUESIPS processing system (Bohlin 1996) and from the NEWSIPS uniform re-processing of the entire archive

\footnotetext{
${ }^{2}$ The numbers are for the total of the archival datasets, although some spectra are for calibration purposes, such as "WAVECAL" or "FLOOD"

${ }^{3}$ http://archive.stsci.edu/iue/
} 
(Nichols \& Linsky 1996). The NEWSIPS version provides a somewhat more photometric product in most cases and uses the optimal extraction algorithm of Kinney et al. (1991). Unless otherwise explicitly stated, all discussion of IUE data refers to the *.MXLO files of NEWSIPS SEDs.

Six WD stars with multiple IUE spectra and with complete CALSPEC UV coverage of the IUE spectral range of 1150-3350 $\AA$ are selected for comparison with their NEWSIPS SEDs, as summarized in Table 1, where the number of good IUE observations appear in the final columns for each IUE camera. The comparisons of our co-adds of the good, low-resolution IUE data in Table 1 with the tabulated CALSPEC SEDs define corrections to the NEWSIPS flux scale. The models, rather than the observations, are the reference SEDs for the primary flux standards G191B2B, GD153, and GD71 that set the HST/STIS flux scale. As part of the co-adding process, each IUE observation is examined for abnormalities, which includes such problems as an exposure too short to produce reasonable signal-to-noise $(\mathrm{S} / \mathrm{N})$, a low or zero signal from a miscentered target acquistion, or solar contamination at the longest wavelengths that happened occasionally near the end of the mission. There are three such problem cases for G191B2B (SWP: 15727, 48543, 55661) and six for HZ21 (SWP: 21845, 21846, 21847, LWP: 02465, 02466, 02472), where these bad observations are not included in the Table 1 counts. The data points that are flagged as bad data quality, such as saturation and reseau marks, are omitted, while the remaining good data points are averaged using the exposure times as weights. The reseau and saturation flagging is somewhat inadequate, so the flags are expanded by one point in each direction. At wavelengths where there are no remaining valid points in the individual co-adds, the assumed flux is a linear interpolation across the gap.

The co-added NEWSIPS fluxes are compared with each CALSPEC star in $20 \AA$ bins for SWP and $40 \AA$ for LWP and LWR to make individual ratios, and then these individual ratios are averaged to derive the global average corrections, as shown in the lowest panels of Figures 113 , The global averages utilize the individual average ratios as weighted by total signal for each star, so the frequently observed G191B2B dominates the results. Also shown in each Figure are the individual residuals for the six stars, i.e. the ratio of the correction derived for each star to the average correction. The average and rms scatter of these residuals appears on the Figures for the

Table 1. Low-Dispersion IUE Data Used for Comparing IUE and CALSPEC SEDs

\begin{tabular}{llcccc}
\hline \hline \multicolumn{1}{c}{ Star } & V (mag) & CALSPEC Name & SWP & LWP & LWR \\
\hline G191B2B & 11.78 & g191b2b_mod_010.fits & 56 & 39 & 10 \\
GD153 & 13.35 & gd153_mod_010.fits & 10 & 8 & 2 \\
GD71 & 13.03 & gd71_mod_010.fits & 10 & 8 & 1 \\
GRW+70 5824 & 12.77 & grw_70d5824_stisnic_007 & 10 & 9 & 1 \\
HZ21 & 14.69 & hz21_stis_004.fits & 17 & 9 & 4 \\
HZ43 & 12.91 & hz43_stis_004.fits & 12 & 1 & 7 \\
\hline
\end{tabular}




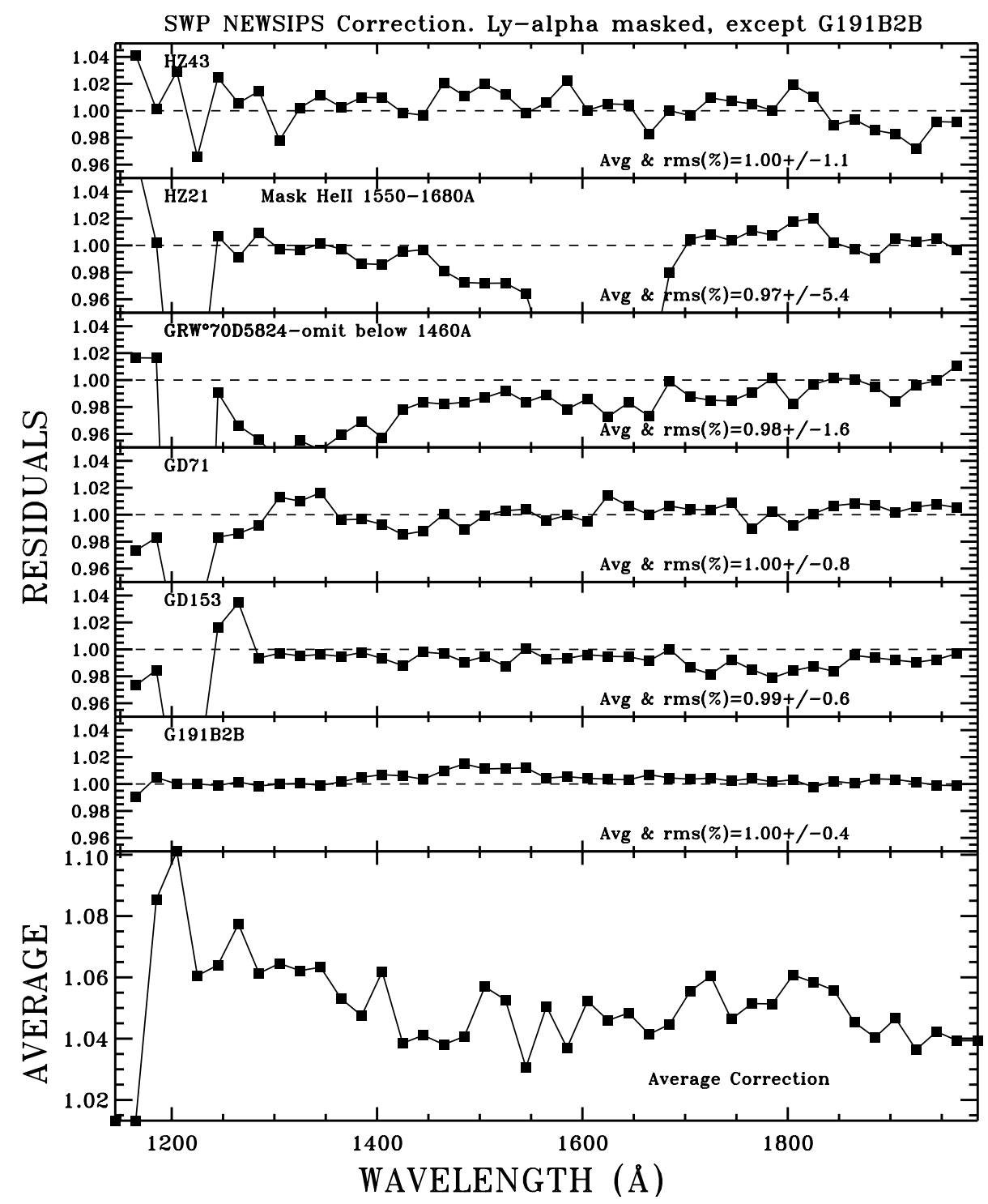

Fig. 1. - Average ratio for the IUE SWP camera of CALSPEC/NEWSIPS fluxes at the bottom and residual ratios for each star, i.e. the residuals show the difference between the correction derived for each star and the average correction ratio. IUE NEWSIPS fluxes should be multiplied by the average corrections of Table 2 to be on the HST/CALSPEC scale. The average and percent rms deviation of the residuals are written for each star for the range of 1300-1900 $\AA$. 


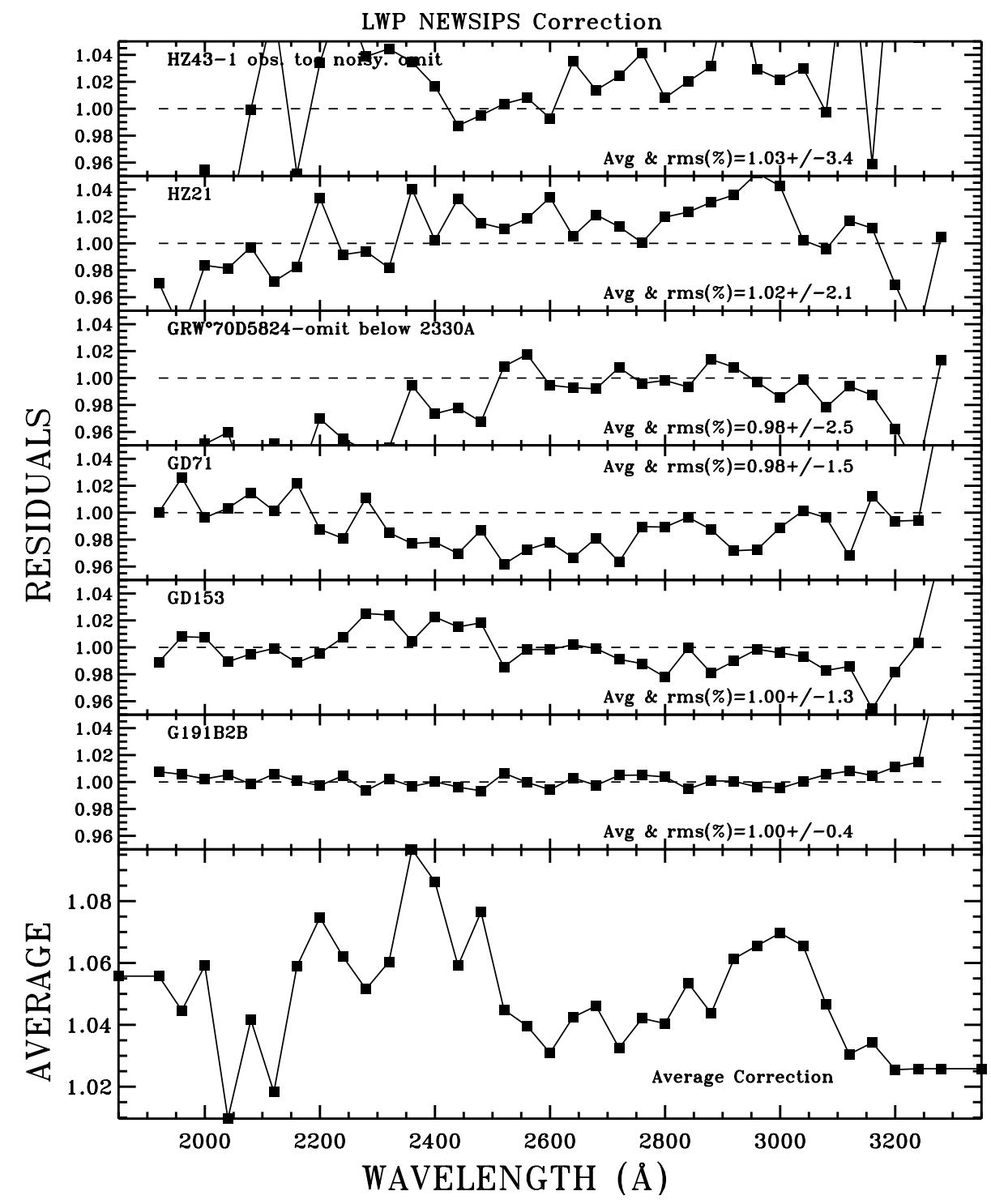

Fig. 2.- Average ratio for the IUE LWP camera of CALSPEC/NEWSIPS fluxes at the bottom and residual ratios for each star for the range of 2100-3000 $\AA$. 


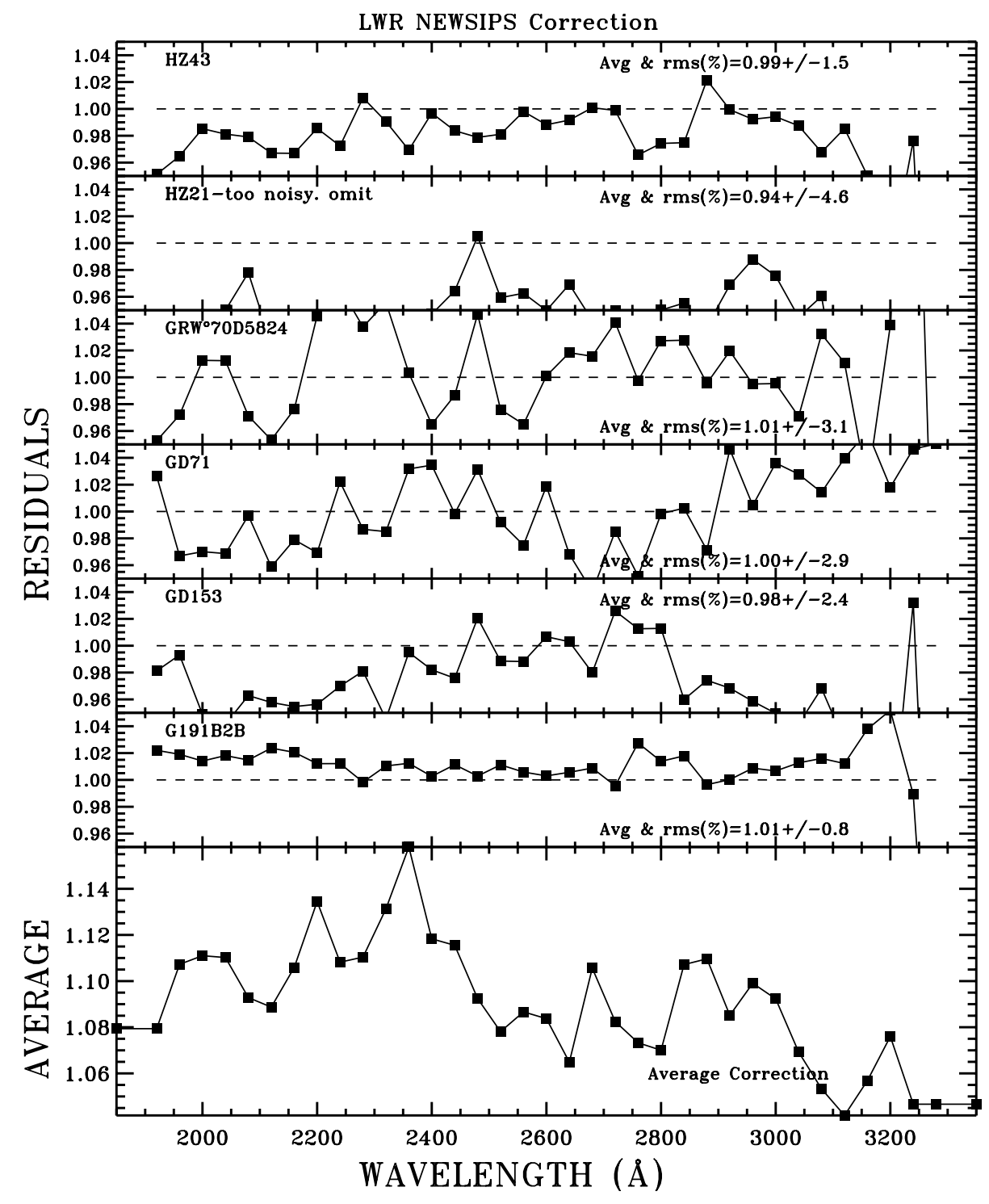

Fig. 3.- Average ratio for the IUE LWR camera of CALSPEC/NEWSIPS fluxes at the bottom and residual ratios for each star for the range of 2100-3000 A. Results for the faintest star HZ21 are often off scale at more than $5 \%$ low. 


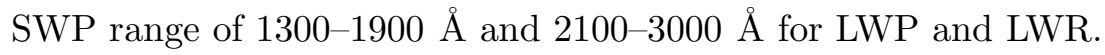

Table 2 contains the final global average of corrections from the six stars, where the regions omitted from these averages are indicated on the plots. The faintest star HZ21 with only four noisy spectra is omitted entirely from the LWR co-add. For the well observed stars, i.e. those with seven or more observations, the separate comparisons with CALSPEC tend to agree fairly well. However, HZ43 is omitted from the SWP average, because the CALSPEC SED uses the slightly less precise FOS data below $1700 \AA$. The global results in Table 2 are extended by one point at each endpoint by the same endpoint bin-value in order to achieve complete wavelength coverage.

Figure 1 shows the best case of SWP, where all six stars are well observed, and all six residuals agree within an uncertainty of $\sim 2 \%$ in the region of higher sensitivity at $1300-1900 \AA$. Near Ly- $\alpha$, the spectra of stars fainter than G191B2B are badly contaminated by geo-coronal Ly- $\alpha$ emission, so the correction near Ly- $\alpha$ is defined by just the one star. Shortward of Ly- $\alpha$, the IUE sensitivity is poor. The omitted anomalous regions for GRW $+70^{\circ} 5824$ and HZ21 are discussed in the next section.

For LWP in the region of higher sensitivity at 2100-3000 $\AA$, five stars are well-observed, and only the faintest star HZ21 has residuals bigger than 4\% in Figure 2. A few GD71 residuals approach 4\%; but for the most part, the brighter stars with the best $\mathrm{S} / \mathrm{N}$ agree better than our conservative estimate of $3 \%$ accuracy for the LWP correction. See the next section for a discussion of the anomalous short wavelength region of the GRW $+70^{\circ} 5824$ spectrum.

For LWR, the data are sparse and only HZ43 with seven co-added spectra provides a worthwhile comparison to G191B2B. Figure 3 shows residuals for HZ43 of less than 3\% at 2100-3000 A, which is our estimate for the precision of the LWR correction in Table 2.

For individual IUE spectra, the repeatability is a function of the exposure level and the background noise, both of which vary with wavelength. The background increases with exposure time and depends on environmental variables, such as the trapped particle flux in the Van Allen radiation belts. However, the rms scatter among the co-added spectra provides a measure of the precision of a typical IUE observation. Figure 4 shows the rms scatter among the G191B2B observations of Table 1 as the solid lines. The SWP repeatability ranges mostly from $4-7 \%$, except at Ly- $\alpha$ and below. The extra scatter at line-center is due to the variability of the contaminating geo-coronal Ly- $\alpha$ emission. LWP repeats best at $\sim 6 \%$ from 2600 to $3000 \AA$ with a loss of precision both shortward and longward. LWR repeats at $8-10 \%$ shortward of $\sim 3000 \AA$ and loses precision even faster than LWP toward longer wavelengths. For our faintest star, HZ21, Figure 5 shows a moderate degradation of precision, where the biggest deviation from the G191B2B results approach a factor of two worse at the shorter wavelengths of LWR; however, there are only four spectra that define the LWR result. The NEWSIPS data files contain estimates for the flux uncertainty, which is propagated to an error-in-the-mean in our co-added and merged files. The IUE NEWSIPS error estimate (sigma) is based on the noise model in the optimal extraction algorithm. The noise model was developed before 1991 and might not be a good representation 
Table 2. Multiplicative Correction for IUE NEWSIPS Fluxes

\begin{tabular}{|c|c|c|c|c|}
\hline Wavelength Å & SWP Correction & Wavelength $\AA$ & LWP Correction & LWR Correction \\
\hline 1145.0 & 1.013 & 1850.0 & 1.056 & 1.079 \\
\hline 1165.0 & 1.013 & 1920.0 & 1.056 & 1.079 \\
\hline 1185.0 & 1.085 & 1960.0 & 1.045 & 1.107 \\
\hline 1205.0 & 1.101 & 2000.0 & 1.059 & 1.111 \\
\hline 1225.0 & 1.061 & 2040.0 & 1.010 & 1.110 \\
\hline 1245.0 & 1.064 & 2080.0 & 1.042 & 1.093 \\
\hline 1265.0 & 1.077 & 2120.0 & 1.018 & 1.089 \\
\hline 1285.0 & 1.061 & 2160.0 & 1.059 & 1.106 \\
\hline 1305.0 & 1.065 & 2200.0 & 1.075 & 1.134 \\
\hline 1325.0 & 1.062 & 2240.0 & 1.062 & 1.108 \\
\hline 1345.0 & 1.063 & 2280.0 & 1.052 & 1.110 \\
\hline 1365.0 & 1.053 & 2320.0 & 1.060 & 1.131 \\
\hline 1385.0 & 1.048 & 2360.0 & 1.097 & 1.158 \\
\hline 1405.0 & 1.062 & 2400.0 & 1.086 & 1.118 \\
\hline 1425.0 & 1.039 & 2440.0 & 1.059 & 1.115 \\
\hline 1445.0 & 1.041 & 2480.0 & 1.077 & 1.092 \\
\hline 1465.0 & 1.038 & 2520.0 & 1.045 & 1.078 \\
\hline 1485.0 & 1.041 & 2560.0 & 1.040 & 1.087 \\
\hline 1505.0 & 1.057 & 2600.0 & 1.031 & 1.084 \\
\hline 1525.0 & 1.053 & 2640.0 & 1.042 & 1.065 \\
\hline 1545.0 & 1.031 & 2680.0 & 1.046 & 1.106 \\
\hline 1565.0 & 1.051 & 2720.0 & 1.033 & 1.082 \\
\hline 1585.0 & 1.037 & 2760.0 & 1.042 & 1.073 \\
\hline 1605.0 & 1.052 & 2800.0 & 1.040 & 1.070 \\
\hline 1625.0 & 1.046 & 2840.0 & 1.054 & 1.107 \\
\hline 1645.0 & 1.048 & 2880.0 & 1.044 & 1.110 \\
\hline 1665.0 & 1.041 & 2920.0 & 1.061 & 1.085 \\
\hline 1685.0 & 1.045 & 2960.0 & 1.066 & 1.099 \\
\hline 1705.0 & 1.055 & 3000.0 & 1.070 & 1.092 \\
\hline 1725.0 & 1.060 & 3040.0 & 1.066 & 1.069 \\
\hline 1745.0 & 1.046 & 3080.0 & 1.047 & 1.053 \\
\hline 1765.0 & 1.051 & 3120.0 & 1.030 & 1.042 \\
\hline 1785.0 & 1.051 & 3160.0 & 1.034 & 1.057 \\
\hline 1805.0 & 1.061 & 3200.0 & 1.025 & 1.076 \\
\hline 1825.0 & 1.058 & 3240.0 & 1.026 & 1.047 \\
\hline 1845.0 & 1.056 & 3280.0 & 1.026 & 1.047 \\
\hline 1865.0 & 1.045 & 3350.0 & 1.026 & 1.047 \\
\hline 1885.0 & 1.040 & & & \\
\hline 1905.0 & 1.047 & & & \\
\hline 1925.0 & 1.036 & & & \\
\hline 1945.0 & 1.042 & & & \\
\hline 1965.0 & 1.039 & & & \\
\hline 1985.0 & 1.039 & & & \\
\hline
\end{tabular}

Note. - Table 2 is published in its entirety in the machine-readable format. 


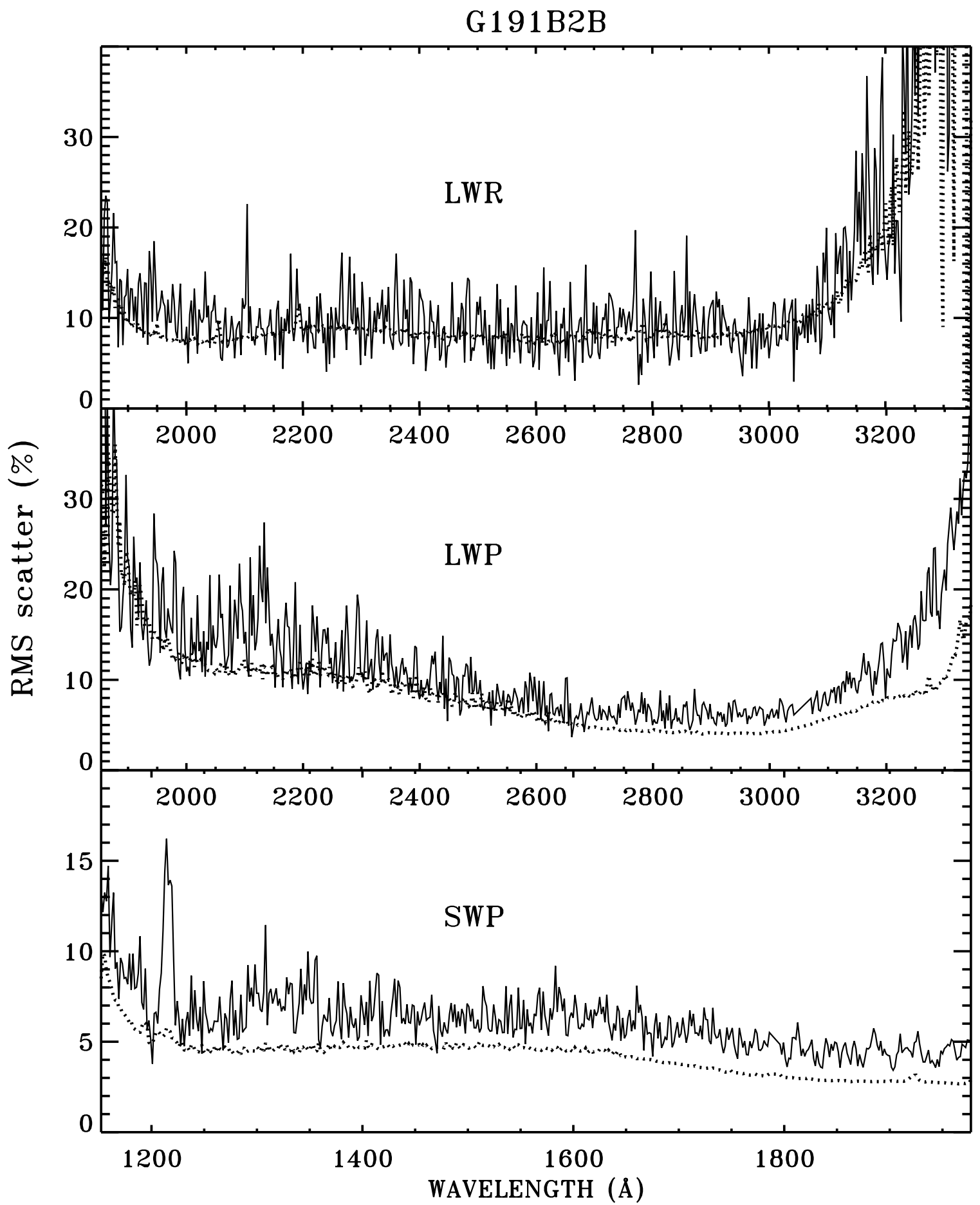

Fig. 4. - Precision of a typical IUE spectrum of G191B2B for each camera, as measured by the rms scatter among the co-added spectra (solid line). The dotted lines are the expected rms scatter as computed from the uncertainty in the flux that is provided in each IUE archival file. These results are for each sample point and would improve with broad band binning. 


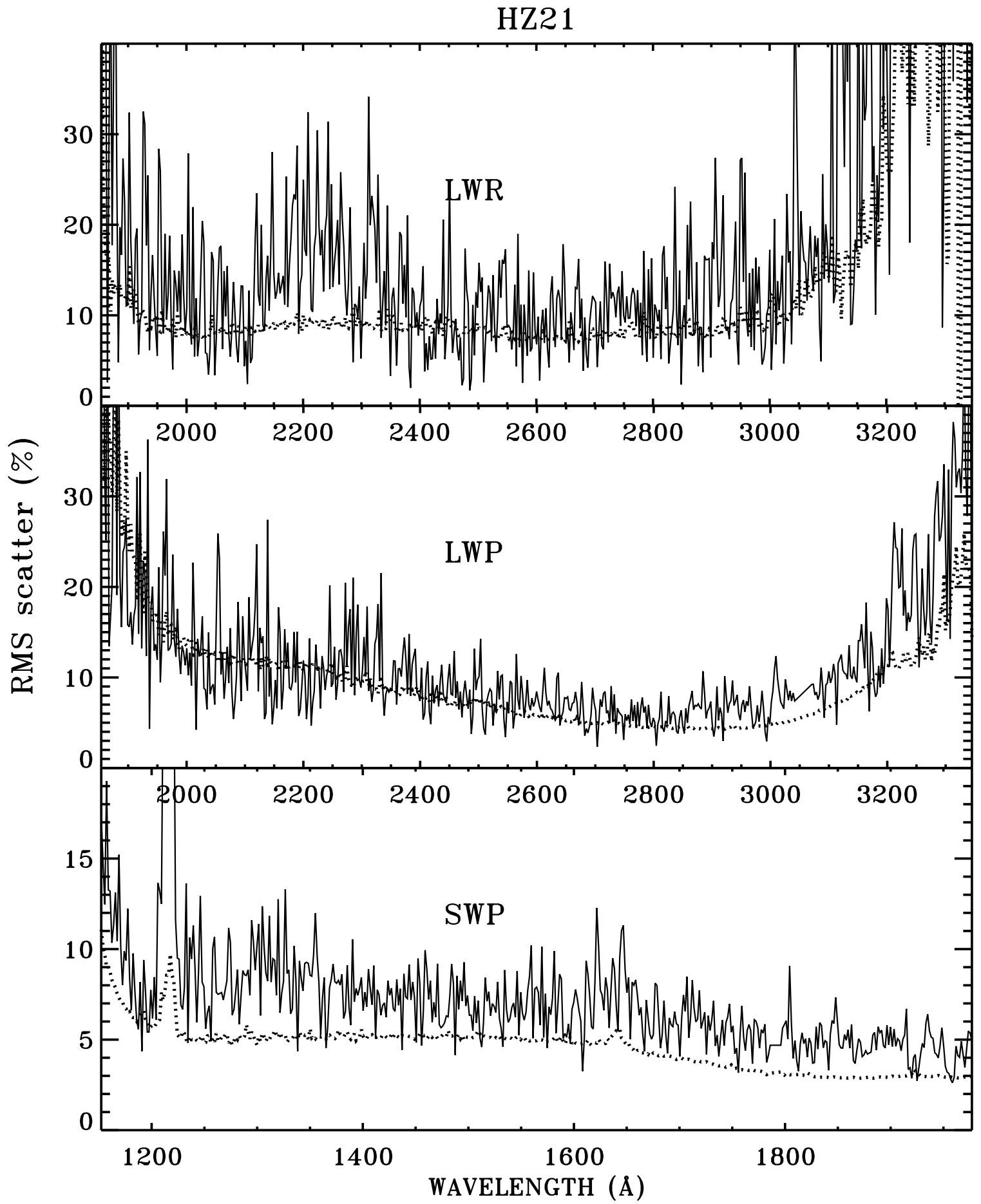

Fig. 5.- Precision of IUE spectra of HZ21 for each camera, as in Figure 4. 
for later observations. Undoubtedly, there are sources of error in addition to the noise model. The dotted lines in Figures 45 are the error-in-the-mean multiplied by the square root of the number of IUE spectra in the co-adds to compare with the solid-line rms scatter. The two uncertainty estimates agree fairly well but with a tendency for the observed rms to be higher, especially for SWP.

\section{Anomalies}

\subsection{H21 $1640 \AA ̊ 丿$ He II}

Apparently, the strong, broad He II line at $1640.5 \AA$ in HZ21 has changed, as illustrated in Figure 6. The 17 IUE spectra all agree within the expectations of Figure 5 and were obtained in the 1978 April-1987 March epoch, while the CALSPEC/STIS data are from 1997 May. The discrepant region from $1550-1680 \AA$ is excluded from the average correction factors. The IUE and STIS disagree by up to $\sim 20 \%$ near the $1640.5 \AA$ line center.

\section{2. $\quad \mathrm{GRW}+70^{\circ} 5824$}

Both SWP and LWP show discrepancies with CALSPEC/STIS at the shorter wavelengths, as shown in Figure 7. The IUE SWP flux is systematically high in the 1250-1500 $\AA$ region by up to $\sim 5 \%$, while LWP is noisy but is in even worse agreement with STIS below $2500 \AA$. However, the single LWR IUE observation shows no discrepancy. An astrophysical cause of the SWP and LWP anomalies is hard to imagine in terms of plausible stellar variability.

The STIS data for GRW $+70^{\circ} 5824$ are from the sensitivity monitoring program with $\sim 90$

separate STIS observations and constitute one of the most precise CALSPEC SEDs. Thus, the anomalous fluxes ratios in the insets of Figure 7 are most likely due to IUE instrumental problems. The LWP flux below $2500 \AA$ shows excess noise, suggesting weak exposure levels in comparison to the background level; and the increasing error toward shorter wavelengths is a signature of a slightly low background subtraction that has a larger effect as the signal drops toward shorter wavelengths. Indeed, the typical background rate per second is more than double that of GD153, while the stellar fluxes are comparable. At $2100 \AA$, the GRW $+70^{\circ} 5824$ flux that is $6 \%$ too high could be caused by only a $\sim 20 \%$ underestimate of the higher than normal background signal.

The anomalous SWP flux for GRW $+70^{\circ} 5824$ is the worst at $1300-1350 \AA$ near the peak of the SWP sensitivity, but our average flux is determined by unsaturated exposures where the background has little effect on the peak net signal. Perhaps, the $\sim 5 \%$ flux errors are just a 2.5 sigma anomaly, or maybe our $2 \%$ uncertainty for the SWP correction is a bit too optimistic. The original IUESIPS fluxes agree with the NEWSIPS averages shown in Figure 7. 


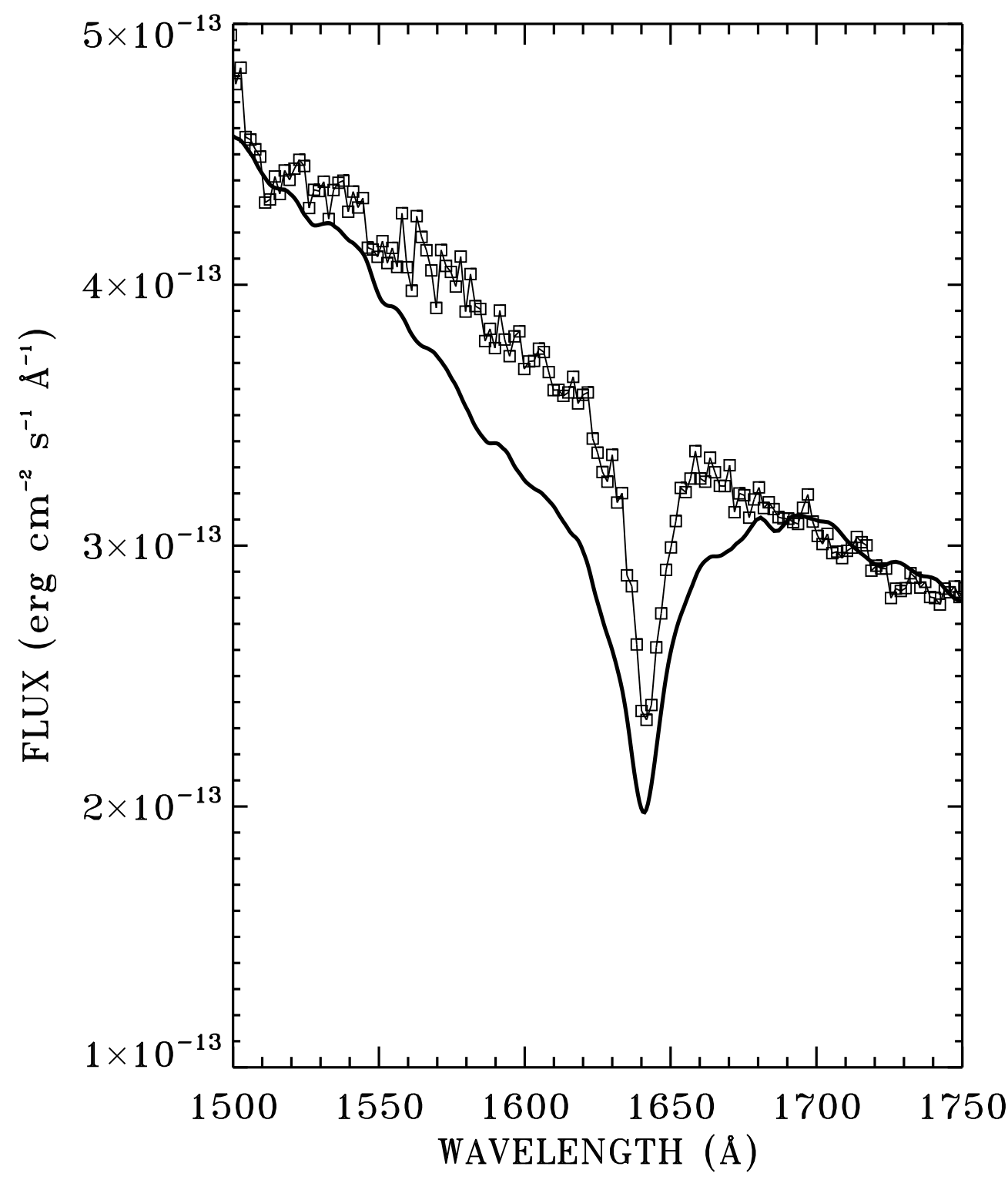

Fig. 6.- Discrepant region of HZ21 near the strong He II $1640.5 \AA$ line. The squares are the average IUE flux from 1978-1987, while the heavy line is the STIS SED from observation o40901nqm in 1997 as convolved to the $6 \AA$ resolution of IUE. 


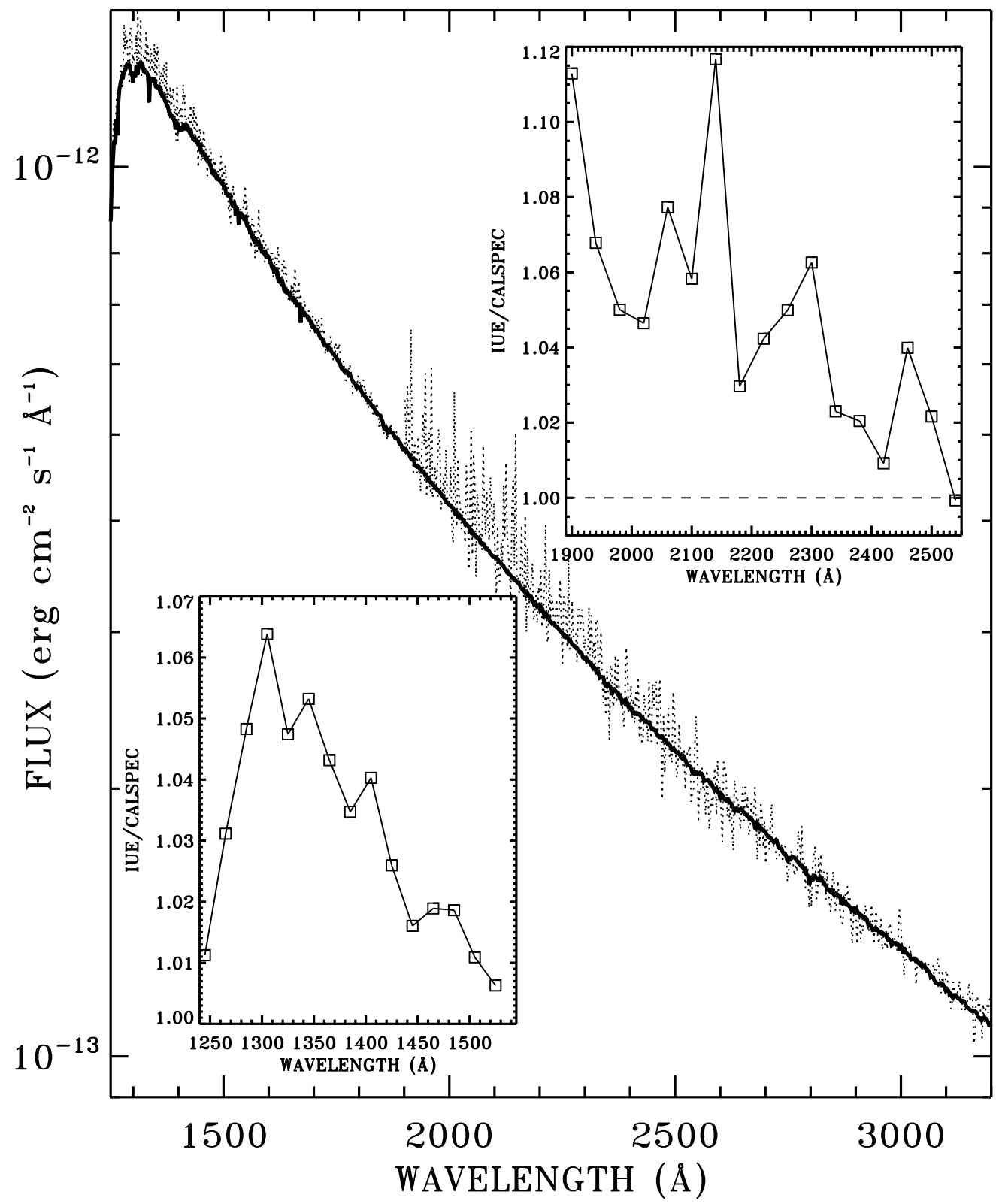

Fig. 7.- Discrepant regions of GRW $+70^{\circ} 5824$ at the shorter wavelengths of SWP and LWP. The dotted lines are the average IUE fluxes for SWP and LWP, while the heavy line is the STIS SED. The inserts show the ratio of the IUE to CALSPEC/STIS in $20 \AA$ bins for SWP and $40 \AA$ bins for LWP in the regions of systematic deviation. 


\section{Electronically Available Results}

The co-added and merged IUE spectra for the six stars of Table 1 and the Table 2 NEWSIPS correction factors are available in the on-line version of this paper and also appear in MAST as High-Level Science Products (HLSP) 4 . The merged ascii files combine all useful datasets as described in Section 2 and cross from SWP to the long wavelength (LW) cameras at $1975 \AA$. These merged files contain eight columns: (1) wavelength in $\AA$, (2) the average net signal in linearized IUE Flux Number (FN) units per second, (3) the average flux in erg $\mathrm{cm}^{-2} \mathrm{~s}^{-1} \AA^{-1}$, (4) the background signal in the same units as the net, (5) the formal propagated uncertainty as the error-in-the-mean in flux units, (6) the number of observations averaged, (7) the total exposure time in seconds, and (8) the rms scatter among the observations in percent. As our planned papers appear, more combined IUE spectra of matched sources having GALEX spectra and the whole collection of GALEX spectra (over 100,000) will also be electronically available. A complete summary of our work along with the machine readable files also appears on our website 5 .

The DOI for the individual IUE spectra from Table 1 is https://dx.doi.org/10.17909/T94M4K, while the DOI for the Tables 28 is https://dx.doi.org/10.17909/T9QD6J.

\section{Summary}

Our comparison of IUE NEWSIPS UV data to the HST-based absolute flux standards in CALSPEC produces a correction factor for the IUE flux scale. The corrections are wavelength-dependent and cover the IUE range of sensitivity from 1150 to $3350 \AA$. The correction of IUE to CALSPEC ranges from factors of 1.01-1.16. IUE-NEWSIPS *.MXLO fluxes should be multiplied by the Table 2 correction factors to be on the HST/CALSPEC flux scale. This new correction will allow more accurate photometric calibration of existing UV datasets, such as GALEX through a comparison with corrected IUE NEWSIPS fluxes, as well as providing critical support for calibration and observation planning of future space missions.

Karen Levy, Bernie Shiao, and Randy Thomson provided many helpful suggestions and clarifications with regard to the IUE MAST database. Scott Fleming entered our HLSP into MAST. Support for this work was provided by NASA through the Space Telescope Science Institute, which is operated by AURA, Inc., under NASA contract NAS5-26555. LB acknowledges support from NASA grant NNX16AF40G. This research made use of the SIMBAD database, operated at CDS,

${ }^{4}$ https://archive.stsci.edu/prepds/iue-fluxcal/

${ }^{5}$ http://dolomiti.pha.jhu.edu/uvsky/iue-fluxcal/ 
Strasbourg, France. 
Table 3. IUE SED for G191B2B

\begin{tabular}{cccccccc}
\hline \hline Wavelength $(\AA)$ & Net $\left(\mathrm{s}^{-1}\right)$ & Flux $^{\mathrm{a}}$ & ${\text { Bkg }\left(\mathrm{s}^{-1}\right)}$ & Sigma $^{\mathrm{a}}$ & No. Obs & ${\text { Exp }\left(\mathrm{s}^{-1}\right)}^{\text {RMS }(\%)}$ \\
\hline 1152.26 & $1.869 \mathrm{e}+00$ & $1.632 \mathrm{e}-11$ & $1.594 \mathrm{e}+00$ & $3.365 \mathrm{e}-13$ & 17.0 & 3562.2 & 13.30 \\
1153.93 & $1.672 \mathrm{e}+00$ & $1.822 \mathrm{e}-11$ & $2.111 \mathrm{e}-01$ & $2.407 \mathrm{e}-13$ & 56.0 & 8208.4 & 12.16 \\
1155.61 & $1.726 \mathrm{e}+00$ & $1.815 \mathrm{e}-11$ & $2.147 \mathrm{e}-01$ & $2.364 \mathrm{e}-13$ & 56.0 & 8208.4 & 13.24 \\
1157.29 & $1.990 \mathrm{e}+00$ & $1.963 \mathrm{e}-11$ & $2.183 \mathrm{e}-01$ & $2.343 \mathrm{e}-13$ & 56.0 & 8208.4 & 12.76 \\
1158.96 & $2.135 \mathrm{e}+00$ & $1.950 \mathrm{e}-11$ & $2.218 \mathrm{e}-01$ & $2.220 \mathrm{e}-13$ & 56.0 & 8208.4 & 14.72 \\
1160.64 & $2.264 \mathrm{e}+00$ & $1.882 \mathrm{e}-11$ & $2.253 \mathrm{e}-01$ & $2.084 \mathrm{e}-13$ & 56.0 & 8208.4 & 9.68 \\
& & & & & & & \\
\hline
\end{tabular}

${ }^{\mathrm{a}} \operatorname{erg~s}{ }^{-1} \mathrm{~cm}^{-2} \AA^{-1}$

Note. - Table 1 is published in its entirety in the machine-readable format. A portion is shown here for guidance regarding its form and content.

Table 4. IUE SED for GD153

\begin{tabular}{cccccccc}
\hline \hline Wavelength $(\AA)$ & Net $\left(\mathrm{s}^{-1}\right)$ & Flux $^{\mathrm{a}}$ & Bkg $_{\left(\mathrm{s}^{-1}\right)}$ & Sigma $^{\mathrm{a}}$ & No. Obs & ${\text { Exp }\left(\mathrm{s}^{-1}\right)}^{\text {RMS }(\%)}$ \\
\hline 1152.26 & $3.454 \mathrm{e}-01$ & $2.946 \mathrm{e}-12$ & $1.932 \mathrm{e}-01$ & $1.617 \mathrm{e}-13$ & 3.0 & 2399.0 & 18.47 \\
1153.93 & $2.943 \mathrm{e}-01$ & $3.301 \mathrm{e}-12-$ & $2.176 \mathrm{e}-03$ & $8.927 \mathrm{e}-14$ & 10.0 & 10196.1 & 9.75 \\
1155.61 & $3.165 \mathrm{e}-01$ & $3.370 \mathrm{e}-12-$ & $1.650 \mathrm{e}-03$ & $8.762 \mathrm{e}-14$ & 10.0 & 10196.1 & 12.24 \\
1157.29 & $3.603 \mathrm{e}-01$ & $3.598 \mathrm{e}-12-$ & $1.125 \mathrm{e}-03$ & $8.707 \mathrm{e}-14$ & 10.0 & 10196.1 & 10.05 \\
1158.96 & $3.866 \mathrm{e}-01$ & $3.543 \mathrm{e}-12-$ & $6.004 \mathrm{e}-04$ & $8.336 \mathrm{e}-14$ & 10.0 & 10196.1 & 8.28 \\
1160.64 & $3.953 \mathrm{e}-01$ & $3.352 \mathrm{e}-12-$ & $7.707 \mathrm{e}-05$ & $8.060 \mathrm{e}-14$ & 10.0 & 10196.1 & 10.27 \\
& & & & & & & \\
\hline
\end{tabular}

${ }^{\mathrm{a}} \mathrm{erg} \mathrm{s}^{-1} \mathrm{~cm}^{-2} \AA^{-1}$

Note. - Table 1 is published in its entirety in the machine-readable format. A portion is shown here for guidance regarding its form and content. 
Table 5. IUE SED for GD71

\begin{tabular}{cccccccc}
\hline \hline Wavelength $(\AA)$ & Net $\left(\mathrm{s}^{-1}\right)$ & Flux $^{\mathrm{a}}$ & ${\text { Bkg }\left(\mathrm{s}^{-1}\right)}^{\text {Sigma }^{\mathrm{a}}}$ & No. Obs & $\operatorname{Exp}_{\left(\mathrm{s}^{-1}\right)}$ & RMS $(\%)$ \\
\hline 1152.27 & $3.000 \mathrm{e}-01$ & $3.272 \mathrm{e}-12$ & $-2.938 \mathrm{e}-02$ & $1.243 \mathrm{e}-13$ & 9.0 & 5781.4 & 19.38 \\
1153.94 & $3.518 \mathrm{e}-01$ & $3.754 \mathrm{e}-12$ & $-3.809 \mathrm{e}-02$ & $1.216 \mathrm{e}-13$ & 10.0 & 6074.9 & 12.48 \\
1155.62 & $3.696 \mathrm{e}-01$ & $3.677 \mathrm{e}-12$ & $-3.746 \mathrm{e}-02$ & $1.168 \mathrm{e}-13$ & 10.0 & 6074.9 & 5.90 \\
1157.29 & $4.070 \mathrm{e}-01$ & $3.817 \mathrm{e}-12$ & $-3.684 \mathrm{e}-02$ & $1.143 \mathrm{e}-13$ & 10.0 & 6074.9 & 8.98 \\
1158.97 & $4.721 \mathrm{e}-01$ & $4.082 \mathrm{e}-12$ & $-3.622 \mathrm{e}-02$ & $1.121 \mathrm{e}-13$ & 10.0 & 6074.9 & 7.68 \\
1160.65 & $4.802 \mathrm{e}-01$ & $3.813 \mathrm{e}-12$ & $-3.559 \mathrm{e}-02$ & $1.069 \mathrm{e}-13$ & 10.0 & 6074.9 & 6.07 \\
& & & & & & & \\
\hline
\end{tabular}

${ }^{\mathrm{a}} \mathrm{erg} \mathrm{s}^{-1} \mathrm{~cm}^{-2} \AA^{-1}$

Note. - Table 1 is published in its entirety in the machine-readable format. A portion is shown here for guidance regarding its form and content.

Table 6. IUE SED for GRW $+70^{\circ} 5824$

\begin{tabular}{cccccccc}
\hline \hline Wavelength $(\AA)$ & Net $\left(\mathrm{s}^{-1}\right)$ & Flux $^{\mathrm{a}}$ & Bkg $_{\left(\mathrm{s}^{-1}\right)}$ & Sigma $^{\mathrm{a}}$ & No. Obs & $\operatorname{Exp}_{\left(\mathrm{s}^{-1}\right)}$ & RMS $(\%)$ \\
\hline 1152.26 & $1.439 \mathrm{e}-01$ & $1.369 \mathrm{e}-12$ & $5.961 \mathrm{e}-02$ & $6.907 \mathrm{e}-14$ & 7.0 & 7413.7 & 11.99 \\
1153.93 & $1.435 \mathrm{e}-01$ & $1.348 \mathrm{e}-12$ & $4.069 \mathrm{e}-02$ & $5.633 \mathrm{e}-14$ & 10.0 & 10580.7 & 7.38 \\
1155.61 & $1.661 \mathrm{e}-01$ & $1.432 \mathrm{e}-12$ & $4.083 \mathrm{e}-02$ & $5.547 \mathrm{e}-14$ & 10.0 & 10580.7 & 12.71 \\
1157.29 & $1.872 \mathrm{e}-01$ & $1.526 \mathrm{e}-12$ & $4.097 \mathrm{e}-02$ & $5.352 \mathrm{e}-14$ & 10.0 & 10580.7 & 9.74 \\
1158.96 & $1.947 \mathrm{e}-01$ & $1.465 \mathrm{e}-12$ & $4.111 \mathrm{e}-02$ & $5.033 \mathrm{e}-14$ & 10.0 & 10580.7 & 5.37 \\
1160.64 & $2.005 \mathrm{e}-01$ & $1.384 \mathrm{e}-12$ & $4.124 \mathrm{e}-02$ & $4.672 \mathrm{e}-14$ & 10.0 & 10580.7 & 7.86 \\
& & & & & & & \\
\hline
\end{tabular}

${ }^{\mathrm{a}} \mathrm{erg} \mathrm{s}{ }^{-1} \mathrm{~cm}^{-2} \AA^{-1}$

Note. - Table 1 is published in its entirety in the machine-readable format. A portion is shown here for guidance regarding its form and content. 
Table 7. IUE SED for HZ21

\begin{tabular}{cccccccc}
\hline \hline Wavelength $(\AA)$ & Net $\left(\mathrm{s}^{-1}\right)$ & Flux $^{\mathrm{a}}$ & ${\text { Bkg }\left(\mathrm{s}^{-1}\right)}$ & Sigma $^{\mathrm{a}}$ & No. Obs & ${\text { Exp }\left(\mathrm{s}^{-1}\right)}^{\text {RMS }(\%)}$ \\
\hline 1152.26 & $1.060 \mathrm{e}-01$ & $9.647 \mathrm{e}-13$ & $5.584 \mathrm{e}-02$ & $2.857 \mathrm{e}-14$ & 14.0 & 28312.8 & 17.37 \\
1153.93 & $1.150 \mathrm{e}-01$ & $9.888 \mathrm{e}-13$ & $5.356 \mathrm{e}-02$ & $2.520 \mathrm{e}-14$ & 16.0 & 34072.3 & 14.81 \\
1155.61 & $1.247 \mathrm{e}-01$ & $1.006 \mathrm{e}-12$ & $5.369 \mathrm{e}-02$ & $2.439 \mathrm{e}-14$ & 16.0 & 34072.3 & 12.47 \\
1157.29 & $1.394 \mathrm{e}-01$ & $1.046 \mathrm{e}-12$ & $5.382 \mathrm{e}-02$ & $2.357 \mathrm{e}-14$ & 16.0 & 34072.3 & 19.28 \\
1158.96 & $1.426 \mathrm{e}-01$ & $9.967 \mathrm{e}-13$ & $5.260 \mathrm{e}-02$ & $2.188 \mathrm{e}-14$ & 17.0 & 35332.1 & 13.27 \\
1160.64 & $1.516 \mathrm{e}-01$ & $9.709 \mathrm{e}-13$ & $5.273 \mathrm{e}-02$ & $2.056 \mathrm{e}-14$ & 17.0 & 35332.1 & 13.20 \\
& & & & & & & \\
\hline
\end{tabular}

${ }^{\mathrm{a}} \operatorname{erg~s}^{-1} \mathrm{~cm}^{-2} \AA^{-1}$

Note. - Table 1 is published in its entirety in the machine-readable format. A portion is shown here for guidance regarding its form and content.

Table 8. IUE SED for HZ43

\begin{tabular}{cccccccc}
\hline \hline Wavelength $(\AA)$ & Net $\left(\mathrm{s}^{-1}\right)$ & Flux $^{\mathrm{a}}$ & Bkg $_{\left(\mathrm{s}^{-1}\right)}$ & Sigma $^{\mathrm{a}}$ & No. Obs & $\operatorname{Exp}_{\left(\mathrm{s}^{-1}\right)}$ & RMS $(\%)$ \\
\hline 1152.26 & $6.090 \mathrm{e}-01$ & $5.383 \mathrm{e}-12$ & $3.758 \mathrm{e}-01$ & $1.635 \mathrm{e}-13$ & 10.0 & 4646.9 & 15.53 \\
1153.93 & $7.200 \mathrm{e}-01$ & $5.756 \mathrm{e}-12$ & $5.555 \mathrm{e}-01$ & $1.532 \mathrm{e}-13$ & 12.0 & 5308.7 & 17.83 \\
1155.61 & $7.274 \mathrm{e}-01$ & $5.510 \mathrm{e}-12$ & $5.559 \mathrm{e}-01$ & $1.470 \mathrm{e}-13$ & 12.0 & 5308.7 & 14.41 \\
1157.29 & $7.897 \mathrm{e}-01$ & $5.528 \mathrm{e}-12$ & $5.562 \mathrm{e}-01$ & $1.470 \mathrm{e}-13$ & 12.0 & 5308.7 & 16.23 \\
1158.96 & $8.672 \mathrm{e}-01$ & $5.614 \mathrm{e}-12$ & $5.566 \mathrm{e}-01$ & $1.388 \mathrm{e}-13$ & 12.0 & 5308.7 & 10.60 \\
1160.64 & $9.610 \mathrm{e}-01$ & $5.716 \mathrm{e}-12$ & $5.569 \mathrm{e}-01$ & $1.342 \mathrm{e}-13$ & 12.0 & 5308.7 & 12.88 \\
& & & & & & & \\
\hline
\end{tabular}

${ }^{\mathrm{a}} \mathrm{erg} \mathrm{s}^{-1} \mathrm{~cm}^{-2} \AA^{-1}$

Note. - Table 1 is published in its entirety in the machine-readable format. A portion is shown here for guidance regarding its form and content. 


\section{REFERENCES}

Boggess, A., Bohlin, R. C., Evans, D. C., et al. 1978, Nature, 275, 377

Bohlin, R. C. 1996, AJ, 111, 1743

-. 2014, AJ, 147, 127

Bohlin, R. C., Dickinson, M. E., \& Calzetti, D. 2001, AJ, 122, 2118

Kinney, A. L., Bohlin, R. C., \& Neill, J. D. 1991, PASP, 103, 694

Kinney, A. L., Calzetti, D., Bohlin, R. C., et al. 1996, ApJ, 467, 38

Nichols, J. S., \& Linsky, J. L. 1996, AJ, 111, 517

Scolnic, D., Rest, A., Riess, A., et al. 2014, ApJ, 795, 45

Wu, C.-C., Schiffer, III, F. H., \& Crenshaw, D. M. 1996, in Astronomical Society of the Pacific Conference Series, Vol. 98, From Stars to Galaxies: the Impact of Stellar Physics on Galaxy Evolution, ed. C. Leitherer, U. Fritze-von-Alvensleben, \& J. Huchra, 130 